\title{
Immune-Mediated Disorders Affecting the Spinal Cord and the Spine
}

\author{
Gina S. Perez Giraldo ${ }^{1}$ • Jorge G. Ortiz Garcia ${ }^{1,2,3}$ (D) \\ Accepted: 17 November 2020 / Published online: 3 January 2021 \\ (C) Springer Science+Business Media, LLC, part of Springer Nature 2021
}

\begin{abstract}
Purpose of Review To review the most recent advances and provide a description of the most common autoimmune diseases causing myelitis and selective spine disorders. The ultimate goal of this article is to facilitate the prompt recognition of these diseases.

Recent Findings The recent discovery of biomarkers such as aquaporin 4 (AQP4) and myelin oligodendrocyte glycoprotein (MOG) antibodies has changed our understanding of autoimmune diseases affecting the spinal cord as well as their treatment and outcomes.

Summary Autoimmune neurology is an increasingly evolving field that encompasses a broad spectrum of autoimmuneinflammatory diseases of the central nervous system (CNS) and peripheral nervous system (PNS). Autoimmune disorders of the spinal cord are a heterogeneous group of myelopathies with a broad differential diagnosis and many of them have been recently identified. Prompt recognition of these myelopathies is important as some of them are treatable, which could improve patient outcomes and prevent disability.
\end{abstract}

Keywords Transverse myelitis · Multiple sclerosis $\cdot$ Neuromyelitis optica spectrum disorder $\cdot$ Myelin oligodendrocyte antibody $\cdot$ Neurosarcoidosis · Paraneoplastic myelitis

\section{Introduction}

Myelopathy refers to any neurological disorder localized to the spinal cord, whereas myelitis implies spinal cord inflammation [1]. The recognition of autoimmune disorders causing myelitis is important as there is a significant potential for disability from these diseases and, furthermore, some of them are treatable. These disorders can have manifestations in other CNS locations, including

This article is part of the Topical Collection on Neurology of Systemic Diseases

Jorge G. Ortiz Garcia

jorge-ortizgarcia@ouhsc.edu

1 Department of Neurology, The University of Oklahoma Health Sciences Center, 920 Stanton L. Young Blvd. Suite 2547, Oklahoma City, OK 73104, USA

2 Division of Critical Care Neurology, The University of Oklahoma Health Sciences Center, 920 Stanton L. Young Blvd. Suite 2547, Oklahoma City, OK 73104, USA

3 Division of Stroke and Cerebrovascular Disorders, The University of Oklahoma Health Sciences Center, 920 Stanton L. Young Blvd. Suite 2547, Oklahoma City, OK 73104, USA the brain and brainstem. This review will focus exclusively in the features of these entities affecting the spinal cord.

Myelitis may result in ascending paralysis, numbness in one or more limbs, and/or sensory level. Severity varies from lack of motor involvement to quadriparesis/quadriplegia. Patients often describe a circumferential band of dysesthesias across the trunk leading to a constricting sensation [2]. Heat-induced worsening of symptoms known as the Uhthoff's phenomenon is characteristic of demyelinating lesions. Patients might also report a shock-like sensation descending down the spine upon neck flexion caused by cervical spine lesions known as Lhermitte's sign. A "sensory useless hand" results from a dorsal column lesion leading to upper extremity proprioception abnormalities [3].

Myelitis may present as an acute or as a chronic progressive condition. Acute transverse myelitis typically evolves over few days with patients reaching their nadir within 28 days [4]. Chronic progressive myelopathy evolves over weeks to months and is the most common presentation of paraneoplastic autoimmune myelopathies and primary progressive multiple sclerosis [3]. 


\section{Immune-Mediated Disorders Affecting the Spinal Cord}

\section{Demyelinating Diseases}

\section{Multiple Sclerosis}

Multiple sclerosis (MS) affects both genders but most commonly presents in women between 20 and 40 years of age. MS can also affect children and the elderly [5]. MS is characterized by excess antibody synthesis within the CNS detectable by measuring oligoclonal bands (OCB) which are elevated in 85 to $90 \%$ of the patients [2] and immunoglobulin $\mathrm{G}$ index (IgG index) in the cerebrospinal fluid (CSF) [5]. MS relapses can affect any part of the CNS and evolve over hours to days with variable severity. MS is the most common cause of short segment myelitis defined as a lesion extending over less than 3 vertebral segments, is often partial causing Brown-Séquard syndrome, and involves the periphery of the spinal cord [3] with predilection for proprioceptive fibers. Clinical findings are often asymmetric [6]. MS rarely presents as longitudinally extensive transverse myelitis (LETM), and if present, it should be considered a red flag for this diagnosis in adults. Children can have a distinct presentation as up to $14 \%$ of cases may have LETM [3, 6].

An acute demyelinating flare will be demonstrated by gadolinium enhancement in the spine MRI usually resolving within 2 to 3 months; $20 \%$ of patients may have ringenhancing spinal cord lesions (Fig. 1a).

Recently, serum or CSF quantification of neurofilament light chain (NfL) has been proposed as a biomarker of axonal damage facilitating disease monitoring [7].

Treatment of acute myelitis due to MS consists of a short course of either intravenous or oral high dose corticosteroids for 3 to 5 days. Steroids improve symptoms but there is no evidence of impact on long-term disability. Many medications have been released in the past 3 decades to reduce relapses and long-term disability. Some require infusions such as natalizumab, ocrelizumab, and ofatumumab which are considered to be the most efficacious. In addition, oral medications like fingolimod, dimethyl fumarate, teriflunomide, and injectables such as interferon beta and glatiramer acetate are now available. The use of vitamin $\mathrm{D}$ has been associated with a lower risk of MS [5]. The diagnostic criteria of MS are beyond the scope of this review article.

\section{Neuromyelitis Optica Spectrum Disorder}

Neuromyelitis optica spectrum disorder (NMOSD) is an inflammatory autoimmune disorder first described in the late nineteenth century as a monophasic disease resulting in simultaneous optic neuritis and transverse myelitis [8, 9]. NMOSD follows a relapsing-remitting course, usually with incomplete recovery between attacks, and patients are often left with considerable disability. The identified etiology is the production of autoantibodies to aquaporin 4 (AQP4) which is the most abundant central nervous system (CNS) water channel and is highly concentrated on astrocytic foot processes at the bloodbrain barrier (BBB) in the floor of the fourth ventricle $[6,7$, 10]. AQP4 IgG is a highly sensitive and specific biomarker which is found in approximately $80 \%$ of the patients [11••]. Incidence of NMOSD ranges from 0.053 to 0.4 per 100,000 people. Asians and African Americans are predominantly affected [9•], with a female predominance of $3: 1$ to $9: 1$ [5]. The disease occurs in both children and adults [8].

Clinical presentation includes recurrent episodes of optic neuritis, often bilateral and involving the posterior optic nerve segment and optic chiasm, and LETM defined as lesions extending over more than 3 vertebral segments. Patients may also present with area postrema syndrome such as intractable nausea, vomiting, and hiccups. This is the result of abundant AQP4 channels in the area postrema. This syndrome is the initial manifestation of NMOSD in about $12 \%$ of patients [9•]. Paroxysmal painful tonic spasms characterized by recurrent stereotyped dystonic-like episodes after an attack of myelitis are also common [3].

NMOSD spinal cord lesions are symmetrical and centrally located and involve the gray matter with extensive spinal cord edema [11••, 12]. Enhancement on MRI is patchy and not very homogeneous with a ring-enhancing appearance in about $30 \%$ of the lesions [7]. In the chronic stage, there is cord atrophy with or without T2 hyperintensity and syrinx-like cavities [12] (Fig. 1b).

Unlike MS, NMOSD has a more inflammatory CSF pattern ( $>50$ nucleated cells) with pleocytosis, hyperproteinorachia, and rare oligoclonal bands [5]. Most patients do not develop a progressive phase [3].

Of note, approximately $14 \%$ of patients with NMOSD present with an initial short lesion which can lead to the misdiagnosis of MS [9•]. The timing of imaging may affect the visualized length of the lesion as imaging too early, within hours to days, may miss a long lesion in evolution, while imaging too late may allow a long lesion to become discontinuous or completely resolved. Enhancement usually resolves in 2 to 3 months [3].

NMOSD frequently coexists with other autoimmune diseases such as systemic lupus erythematosus (SLE), Sjögren, autoimmune thyroid disease, type 1 diabetes mellitus, and ulcerative colitis $[2,11]$. Some patients with SLE have positive AQP4 antibodies; the current consensus states that these are separate diseases that coexist [1]. NMOSD may also result from paraneoplastic syndromes, most commonly associated with breast cancer, lung cancer, cervical cancer, thymoma, and lymphoma $[3,6,13,14]$. Treatment of acute attacks is with high-dose intravenous corticosteroids [11 • ]. Plasmapheresis (PLEX) every other day for 5 to 7 sessions 

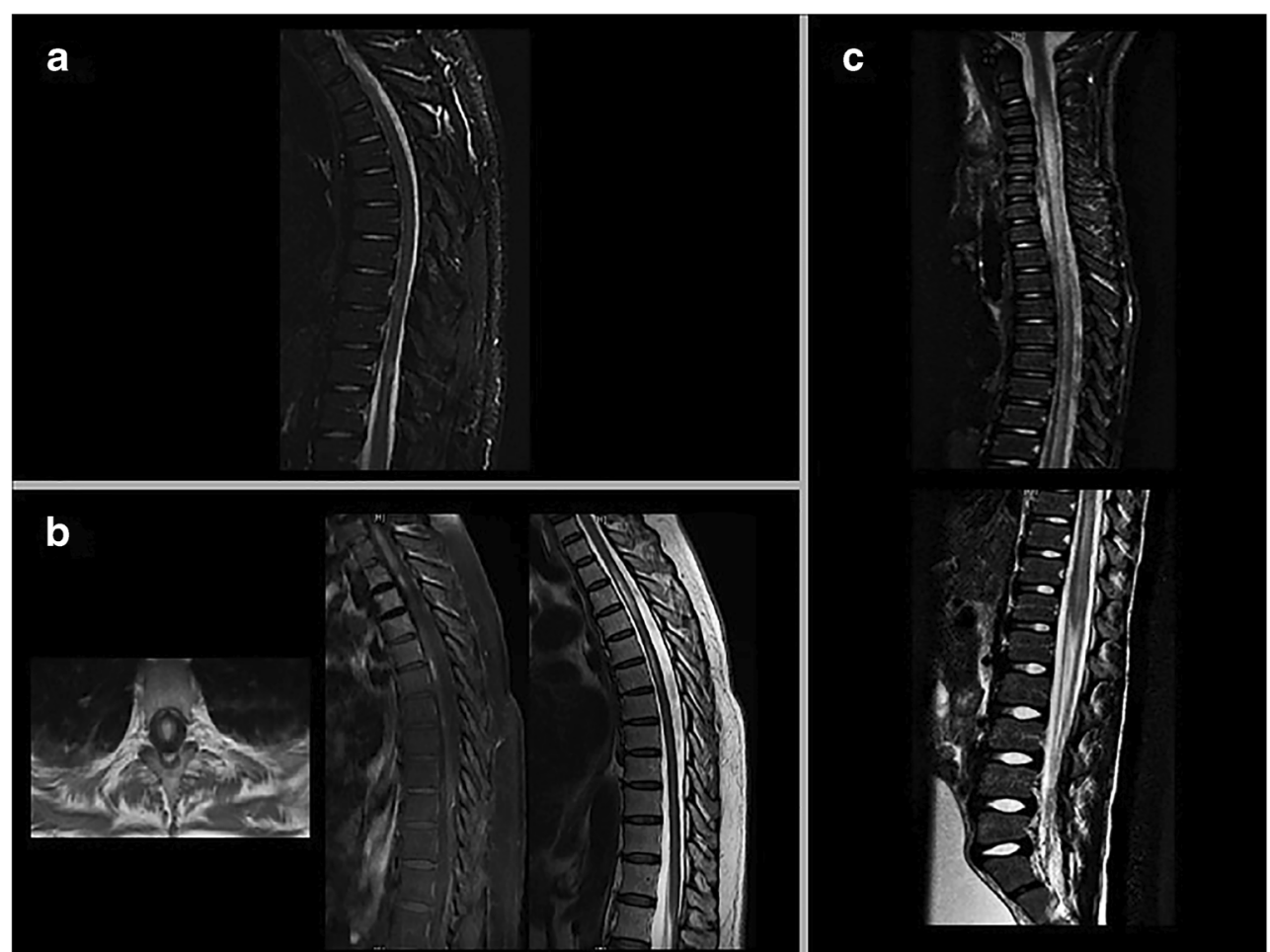

Fig. 1 a Sagittal spine MRI T2/STIR sequence shows increased signal along the anterior aspect of the spinal cord at $\mathrm{T} 9$ and dorsal aspect at $\mathrm{T} 11$ in a patient with MS myelitis. b Axial and sagittal spine MRI T1 postcontrast demonstrates enhancing lesions in the central spinal cord at T2T4 and T5-T6. Also, a T2/STIR sequence shows signal abnormalities in the same regions consistent with NMOSD. c Sagittal spine MRI T2 FLAIR sequence demonstrates signal changes in the gray matter from

is used with or after steroid therapy in those whose symptoms did not improve or who have a severe clinical syndrome [9•]. Long-term use of immunomodulators include azathioprine, mycophenolate mofetil, rituximab, and the recently FDAapproved monoclonal antibodies eculizumab, inebiluzumab, and satralizumab $[5,15]$. The use of Interferon beta-1a has been associated with marked worsening of NMOSD [2] as interferon beta exacerbates lymphocyte T helper-17 (Th17). The Th17 increases the inflammatory response in NMOSD [16].

\section{Myelin Oligodendrocyte Glycoprotein IGG Associated Disease (MOGAD)}

Myelin oligodendrocyte glycoprotein (MOG) is a glycoprotein located on the myelin surface and found exclusively in the central nervous system (CNS) [17]. In the past, MOG antibodies were thought to be involved in multiple sclerosis [18]. It is now known that MOGAD is a distinct entity with distinct pathophysiology and phenotypic features. MOGAD has been associated with severe demyelination without astrocytic cell loss usually observed in AQP4 positive patients. Prevalence is higher among children. Men are more frequently affected [19]. Clinical presentation varies with age. Young children the cervical spine extending caudally to the conus medullaris in a patient with ADEM with positive MOG antibodies. Image 1c courtesy of Dr. Cherie Herren. MRI, magnetic resonance imaging; STIR, short-TI inversion recovery; FLAIR, fluid-attenuated inversion recovery; ADEM, acute disseminated encephalomyelitis; MOG, myelin oligodendrocyte glycoprotein; MS, multiple sclerosis

typically present with acute disseminated encephalomyelitis (ADEM), whereas children older than 9 years old and adults present with extensive optic neuritis, often bilateral with perineural enhancement, longitudinally extensive transverse myelitis (LETM) that is often but not exclusively longitudinally extensive and brainstem lesions [19,20]. Conus medullaris syndrome is characteristic of MOGAD [7]. (Fig. 1c).

It has been hypothesized that the higher presence of MOG antibodies in children could represent an age-dependent manifestation of demyelinating diseases [20].

MOG-IGG-associated myelitis has been reported to be mostly monophasic; relapses might occur but those appear to be less frequent and have a better outcome as compared to AQP4 positive NMOSD patients [3, 19]. Serum MOG antibodies should be repeated after 6 months and 1 year to identify patients with persistent antibodies as those have a higher risk to develop a relapse with poor recovery [20], whereas patients with transient seropositivity are more likely to have a monophasic presentation $[11 \bullet \cdot$. CSF demonstrates pleocytosis with more than 50 white blood cells; oligoclonal bands are unusual. It is not recommended to measure MOG antibodies in CSF as levels are typically low and only cellbased assays should be used to identify MOG antibodies in serum $[17,18,20]$. 
Treatment of the acute phase is with high-dose intravenous glucocorticoids for 3 to 5 days. PLEX and intravenous immunoglobulin therapy (IVIG) can be used if there is no improvement with intravenous steroids [17]. Steroids should be tapered slowly as there is a high risk of flare-ups within 2 months of discontinuation, prednisone doses less than $10 \mathrm{mg}$ per day, or with rapid taper $[18,21]$. Long-term management is controversial as the disease may be monophasic. There are limited data on immunotherapy in patients with MOG antibodies; some authors suggest monthly IVIG infusions, mycophenolate mofetil, or rituximab in the event of relapsing disease [20, 21].

\section{Acute Disseminated Encephalomyelitis}

This is an immune-mediated disease seen primarily in children and characterized by widespread simultaneous CNS demyelination leading to multifocal neurological symptoms. Generally, ADEM follows a monophasic course, and in about two-thirds of cases, there is a preceding infection or vaccination within 4 weeks of the onset of symptoms [5, 8]. Main clinical manifestations include encephalopathy, optic neuritis, transverse myelitis, seizures, or coma [8]. Myelitis occurs in approximately $24 \%$ of the patients [2], the thoracic spinal cord is predominantly affected, and lesions are of similar age and display a homogenous gadolinium enhancement pattern [2, 5]. Brain MRI shows large multifocal white matter lesions and deep gray matter lesions that frequently involve the basal ganglia and thalami $[11,22]$. Typically, there is prominent CSF pleocytosis, hyperproteinorachia, but no oligoclonal bands [2]. MOG antibodies have been found in children with ADEM [20] Fig. 1c.

Treatment of ADEM is with high-dose corticosteroids followed by a prolonged oral taper over weeks as relapse during steroid taper is common $[3,6]$. If there is a progression of symptoms with steroids, IVIG, and plasmapheresis can be considered [5]. Long-term treatment is not required [6].

\section{Glial Fibrillary Acidic Protein Astrocytopathy (GFAP-IGG)}

GFAP astrocytopathy refers to an inflammatory CNS autoimmune disease associated with GFAP antibodies in the CSF. Meningoencephalitis is the most common presentation leading to subacute or chronic encephalopathy. Patients may also present with seizures, headaches, psychiatric symptoms, and myelitis. GFAP-IGG occurs at any age; children account for $10 \%$ of cases [23]. There is an association with ovarian teratomas suggesting a paraneoplastic pathophysiology [11••].

Brain MRI with contrast demonstrates a characteristic linear, radial perivascular pattern of white matter enhancement. Patients may also have leptomeningeal enhancement [11••]. There is LETM on spine MRI with a central cord enhancement on T1 sagittal images [23]. Isolated myelitis does not occur. CSF demonstrates pleocytosis with lymphocytic predominance and hyperproteinorachia, and half of the patients may have oligoclonal bands $[11 \bullet \bullet$. Treatment is with corticosteroids with rapid clinical and radiological improvement, although about 20 to $50 \%$ of patients can have a relapsing disease requiring chronic immunotherapy with mycophenolate mofetil, azathioprine, rituximab, or cyclophosphamide [23].

\section{Collapsin Response Mediator Protein-5 Autoantibody (CRMP-5)}

CRMP-5 IGG autoantibodies can lead to autoimmune neurological symptoms. Patients with CRMP-5 may present with optic neuropathy, retinitis, chorea, ataxia, neuropathy, and myelitis. This disease has predilection for children and occurs as a paraneoplastic or post infectious phenomenon. Transverse myelitis tends to be longitudinally extensive involving lateral or dorsal columns [11, 24, 25].

\section{Systemic Autoimmune Disorders}

Systemic autoimmune diseases can affect the CNS causing myelitis. We summarize the most common systemic disorders involving the spinal cord.

\section{Sarcoidosis}

Sarcoidosis is a granulomatous disorder that can affect every organ system. Incidence has been estimated to be 10.9 to 35.5 cases per 100,000. More prevalent among African Americans [8], neurosarcoidosis occurs in 5 to $15 \%$ of patients with sarcoidosis. Isolated neurosarcoidosis occurs in approximately $1 \%$ of patients. A history of erythema nodosum, lymphadenopathy, or anterior uveitis are clues to the diagnosis of sarcoidosis [6].

Neurosarcoidosis may present with a progressive myelopathy, which does not follow a typical relapsing-remitting course observed with some demyelinating disorders. Relapses may occur while weaning immunotherapy [3]. Spinal cord involvement in sarcoidosis has a predilection for the thoracic spinal cord. Patients often have a LETM with intense homogeneous subpial enhancement extending inwards from the dorsal cord with central canal enhancement on sagittal images; this is known as the "trident sign" which is characteristic of this disease [12]. Lesions may be multifocal, and spinal cord enhancement can persist for many months. Ring enhancing lesions are not typically seen [2, 6, 7]. CSF is generally inflammatory with lymphocytic pleocytosis, hypoglycorrhachia, and infrequent OCB. Serum soluble interleukin-2 receptor (sIL-2R) is a sensitive biomarker in the diagnosis of sarcoidosis and has superior sensitivity $(88 \%)$ and specificity (85\%) compared to ACE [26]. 
Angiotensin-converting enzyme (ACE) is an insensitive marker and nonspecific for the diagnosis [2, 5].

\section{Immunoglobulin G4-Related Disease}

Immunoglobulin G4-related disease (IGG4-RD) is a fibroinflammatory systemic disease of unknown cause affecting any organ and the cause of inflammatory tumefactive lesions. Prevalence of IGG4-RD seems to be higher among men within the sixth or seventh decades of life [27, 28]. CNS manifestations of IGG4-RD most commonly include hypophysitis and hypertrophic pachymeningitis [29]. IGG4$\mathrm{RD}$ can also result in myelopathies due to an inflammatory pseudotumor causing compression of the spinal cord [30].

Diagnosis of IGG4-RD depends on the presence of increased numbers of IGG4 positive plasma cells in tissue infiltrates, in addition to specific histopathological features. Key histopathological features include the following: (1) dense lymphoplasmacytic infiltrates, (2) storiform pattern of fibrosis, and (3) obliterative phlebitis. Serum IGG4 level is not a sensitive diagnostic marker as is normal in 25-30\% of patients with confirmed IGG4-RD. First line of treatment is with glucocorticoids. Typically, patients have a good response. However, relapse rates during steroid withdrawal are high. If untreated, IGG4-RD can progress to extensive fibrosis [30, 31].

\section{Systemic Lupus Erythematous}

Myelitis, one of the 19 neuropsychiatric syndromes related to systemic lupus erythematous (SLE) occurs only in 1 to $2 \%$ of cases within the first 5 years after disease onset [2]. The pathogenesis may be related to arterial thrombosis and smallvessel vasculitis leading to axonal and neural damage (1). SLE-related myelitis patients often have high titers of ANA, anti-double-stranded DNA antibodies, and hypocomplementemia (2). Onset is acute and progresses over hours or days leading to LETM that in severe cases can extend rostrally into the medulla. Neurological manifestations may be preceded by general symptoms such as fever, headache, and vomiting. Thoracic cord segments are mostly involved. Several reports indicate an important association between antiphospholipid antibodies, and myelopathy. Treatment is with IV methylprednisolone and cyclophosphamide. Recurrence is common and can occur within a year in up to $50 \%$ of patients $[1,2]$.

\section{Antiphospholipid Syndrome}

Antiphospholipid syndrome is a systemic, autoimmune disorder characterized by recurrent thrombotic events mediated by antiphospholipid antibodies. Myelitis is an unusual presentation of antiphospholipid syndrome (APS), and frequency ranges between 0.4 and $4 \%$ patients [34].

\section{Behcet's Disease}

Behcet's disease is a chronic relapsing multisystemic inflammatory disorder of unknown etiology. The disease is more common in eastern Mediterranean countries. Neurological manifestations can precede systemic manifestations [32]. Classic presentation includes oral ulcers, genital ulcers, uveitis, and pathergy [11••]. The nervous system may be involved in $9.4 \%$ of the cases and typically occurs in the third or fourth decade of life [8]. Myelitis occurs in up to one-third of neuro Behcet's cases with a predilection for the cervical and thoracic spinal cord causing LETM with increased T2 signals in the intramedullary region. HLA-B51 is the strongest genetic susceptibility factor for Behcet's disease [33], and genetic testing may be helpful to support the diagnosis [32].

\section{Sjögren Syndrome}

Sjögren syndrome is a chronic autoimmune disorder more commonly among middle age to elderly women. Neurologic manifestations may be the presenting symptoms of this disease in up to two-third of patients. Anti-RO/SSA antibodies have been associated with recurrent myelitis. Sjögren-associated myelitis frequently involves the cervical spinal cord and can be longitudinally extensive. Treatment with IV cyclophosphamide or rituximab has been reported to be effective [2].

\section{Vogt-Koyanagi-Harada Disease}

This is an idiopathic, systemic autoimmune disorder that affects melanin forming cells. The disease can result in bilateral uveitis, alopecia, poliosis, vitiligo, and deafness with transverse myelitis being a rare manifestation of this disease. Treatment is with steroids [35].

\section{Paraneoplastic Disorders}

Paraneoplastic myelitis usually occurs in the setting of multifocal paraneoplastic neurological disorders. Isolated paraneoplastic myelopathies are frequently misdiagnosed as primary progressive MS [3]. In most cases, the neurological symptoms precede the detection of cancer and the diagnosis is not excluded by failure to detect antibodies [6]. These myelopathies have a chronic presentation and are most commonly associated with lung and breast carcinomas [3].

CSF shows a lymphocytic pleocytosis (usually $<100$ cells) with hyperproteinorachia. Spine MRI demonstrates a classic longitudinally extensive T2 signal abnormality confined to specific tract (lateral and dorsal column) that may enhance 
symmetrically with gadolinium leading to a classic "owl eye" appearance in the axial view. In $50 \%$ of the cases, the spine MRI can be normal [6].

Neural autoantibodies that predict cancer most strongly recognize intracellular onconeural proteins. In contrast, autoantibodies targeting neuronal and glial plasma membrane proteins are less strongly associated with cancer. Amphiphysin and collapsing response-mediator protein type 5 (CRMP 5) immunoglobulin G are the most common neural autoantibodies associated with paraneoplastic myelopathies [3].

The treatment of paraneoplastic myelopathies is to first treat the underlying cancer causing the autoantibodies with either surgery, radiation, or chemotherapy. Immunotherapy is also used to decrease the progression of neurological impairment. It is suggested to use corticosteroids, IVIG, and cyclophosphamide as treatment. If improvement occurs, immunotherapy should be continued [6] Table 1.

\section{Stiff-Person Syndrome}

Stiff-person syndrome is caused by glutamic acid decarboxylase (GAD) antibodies, and anti-amphiphysin antibodies which are commonly associated with a paraneoplastic phenomenon. Stiff-person syndrome (SPS) is characterized by truncal rigidity and proximal muscle spasms. SPS may occur in conjunction with transverse myelitis as anti-amphiphysin antibodies can attack the spinal cord [36].

\section{Autoimmune/Paraneoplastic Motor Neuron Disorders}

Rarely, motor neuron disease may be a manifestation of a paraneoplastic myelopathy associated with lung, breast, renal, or hematologic malignancies. Autoantibodies associated with this condition include ANNA-1 (anti-Hu), Purkinje cell antibody type 1 (anti-Yo) [6].

\section{Parainfectious Autoimmune Myelopathies}

Myelopathies may be preceded by infections triggering an autoimmune reaction due to molecular mimicry. It follows a monophasic course that is steroid responsive. Triggering infections typically have resolved before onset of myelitis. Most commonly implicated organisms include hepatitis $\mathrm{C}$ virus, Mycoplasma pneumoniae, herpes zoster, dengue virus, Zika virus, Campylobacter jejuni, and Enterobius vermicularis [2, $37,38]$. Cases of acute transverse myelitis secondary to severe acute respiratory syndrome coronavirus 2 (SARS-CoV-2) infection have been reported in adults and children [44-49].

\section{Immune-Mediated Disorders Affecting the Spine}

\section{Ankylosing Spondylitis}

Ankylosing spondylitis (AS) is a chronic, inflammatory disease of the axial skeleton leading to bone loss and erosions with subsequent new bone formation [39].

Table 1 Antibodies causing Paraneoplastic Myelitis or Motor Neuron Disease

\begin{tabular}{|c|c|c|}
\hline Antibody & Clinical presentation & Related cancer \\
\hline Amphiphysin & $\begin{array}{l}\text { Myelitis } \\
\text { Stiff-person syndrome }\end{array}$ & Small cell lung cancer \\
\hline CRMP 5 & $\begin{array}{l}\text { Myelitis } \\
\text { Neuropathy } \\
\text { Ataxia }\end{array}$ & Small cell lung cancer \\
\hline Aquaporin 4 antibodies & $\begin{array}{l}\text { Transverse myelitis } \\
\text { Optic neuritis } \\
\text { Area postrema syndrome }\end{array}$ & $\begin{array}{l}\text { Lung cancer } \\
\text { Breast cancer } \\
\text { Cervical cancer } \\
\text { Thymoma } \\
\text { Lymphoma }\end{array}$ \\
\hline Anti-Hu & Motor neuron disease & $\begin{array}{l}\text { Hematologic malignancies } \\
\text { Lung cancer } \\
\text { Renal cancer } \\
\text { Ovary cancer }\end{array}$ \\
\hline Anti-Yo & Motor neuron disease & $\begin{array}{l}\text { Hematologic malignancies } \\
\text { Lung cancer } \\
\text { Renal cancer } \\
\text { Ovary cancer }\end{array}$ \\
\hline
\end{tabular}


Patients with AS are prone to fracture with minimal injury. AS has been associated with the HLA-B27 gene and is more common among men [40]. Patients with AS are more susceptible to vertebral fractures mostly in the lower cervical spine. Fractures are often unstable and can lead to neurological complications such as spinal cord injury, nerve root injury, spinal epidural hematomas, and spinal stenosis. MRI of the spine is the method of choice to rule out spinal cord injury [39].

\section{Rheumatoid Arthritis}

Rheumatoid arthritis (RA) is a systemic autoimmune disease resulting in chronic synovial inflammation. RA commonly involves the cervical spine within the first 2 years of the disease in most of the patients. Atlantoaxial subluxation is a feared complication which can lead to cervical spine instability, with subsequent severe neurological complications such as motor or sensory deficits [41]. Spinal cord compression is the leading cause of sudden death in patients with RA [42].

\section{Systemic Juvenile Idiopathic Arthritis}

This is the most common autoimmune disease of connective tissue in childhood causing progressive joint degeneration. Patients can present with fever, erythematous rash, lymphadenopathy, serositis, and arthritis. The spine can be affected causing synovial joint inflammation with neurological complications such as spinal canal stenosis and atlantoaxial subluxation [43].

\section{Conclusions}

General neurologists and neurohospitalists should be aware that autoimmune disorders affecting the spinal cord are a heterogeneous group of myelopathies and an evolving field in Neuroimmunology during the last two decades. Clinically, the primary feature of many of these entities is the inflammatory process of the spinal cord known as myelitis. The similarities in the clinical presentation and the variety of associated conditions could cause confusion at the time of diagnosis. Some unique features of transverse myelitis can help in the recognition and differentiation of these diseases. The longitudinally extensive form is found mainly in NMO SD, sarcoidosis, and MOG-IGG-associated disease whereas MS most commonly presents as short segment myelitis.

Several biomarkers are helpful in the understanding of the pathophysiological mechanisms and targeted treatments. One of the most recent discovered markers, the myelin oligodendrocyte glycoprotein antibodies, has allowed us to differentiate MOG-IGG-associated disease from NMO-SD and MS. CSF studies have been described in each entity previously, but in general, these conditions demonstrate pleocytosis with lymphocytic predominance and hyperproteinorachia. Dedicated spine MRI should include gadolinium injection to enhance active lesions and should be done in all patients with myelopathy. Finally, the prompt recognition of these diseases and the immediate treatment prevent disability and assure better outcomes. The long-term care should include a follow-up with a neuroimmunologist, and physical therapy if the patient remains with neurological deficits.

\section{Compliance with Ethical Standards}

Conflict of Interest The authors declare that they have no conflict of interest.

Human and Animal Rights and Informed Consent This article does not contain any studies with human or animal subjects performed by any of the authors.

\section{References}

Papers of particular interest, published recently, have been highlighted as:

- Of importance

•- Of major importance

1. Chiganer EH, Hryb JP, Carnero Contentti E. Myelitis and lupus: clinical manifestations. Diagn Treat Rev Reumatol Clin. 2017;13(6):344-8.

2. Beh SC, Greenberg BM, Frohman T, Frohman EM. Transverse myelitis. Neurol Clin. 2013;31:79-138.

3. Flanagan EP. Autoimmune myelopathies. Handb Clin Neurol. 2016;133:327-51.

4. Greenberg BM, Krishnan C, Harder L. New onset transverse myelitis diagnostic accuracy and patient experiences. Mult Scler Relat Disord [internet]. 2019;30(January):42-4. Available from. https:// doi.org/10.1016/j.msard.2019.01.046.

5. Galetta KM, Bhattacharyya S. Multiple sclerosis and autoimmune neurology of the central nervous system. Med Clin North Am [Internet]. 2019;103(2):325-36. Available from. https://doi.org/ 10.1016/j.mena.2018.10.004.

6. Flanagan EP. Autoimmune myelopathies. Contin Lifelong Learn Neurol. 2011;17(4):776-99.

7. Ciccarelli O, Cohen JA, Reingold SC, Weinshenker BG, Amato MP, Banwell B, et al. Spinal cord involvement in multiple sclerosis and neuromyelitis optica spectrum disorders. Lancet Neurol. 2019;18(2):185-97.

8. Sung-Min K, Seong-Joon K, Haeng JL, Hiroshida K, Palace J, Fujihara K. Differential diagnosis of neuromyelitis optica spectrum disorders. Ther Adv Neurol Disord. 2017;10(7): 265-89.

9. Pittock SJ, Lucchinetti CF. Neuromyelitis optica and the evolving spectrum of autoimmune aquaporin-4 channelopathies: a decade later. Ann N Y Acad Sci. 2016;1366(1):20-39 This is an extensive review of the clinical evidence in neuromyelitis optica and the spectrum of this entity. The article describes a detailed synthesis of contemporary issues in this topic.

10. Bernard-Valnet R, Cobo-Calvo A, Siegfried A, Marasescu R, Bonnan M, Ballan G, et al. Paraneoplastic neuromyelitis optica and ovarian teratoma: a case series. Mult Scler Relat Disord 
[Internet]. 2019;31(March):97-100. Available from. https://doi. org/10.1016/j.msard.2019.03.031.

11.• Flanagan BEP. By Eoin P. Flanagan, MBBCh. Neuromyelitis optica spectrum disorder and other CNS inflammatory diseases. Continuum (N Y). 2019:815-44 The most extensive and updated review of central nervous system inflammatory diseases. The authors develop clinical scenarios of patients and guide in how to diagnose the specific condition. The article includes an useful summary of the current status of immune-mediated disorders affecting the spinal cord.

12. Geraldes R, Ciccarelli O, Barkhof F, De Stefano N, Enzinger C, Filippi M, et al. The current role of MRI in differentiating multiple sclerosis from its imaging mimics. Nat Rev Neurol [Internet]. 2018;14(4):199-213. Available from. https://doi.org/10.1038/ nrneurol.2018.14.

13. Zekeridou A, Lennon VA. Aquaporin-4 autoimmunity. Neurol Neuroimmunol NeuroInflamm. 2015;2(4):1-10.

14. Yuan J, Jia Z, Qin W, Hu W. Paraneoplastic neuromyelitis optica spectrum disorder associated with breast cancer. Clin Interv Aging. 2019;14:1039-44.

15. Holmøy T, Høglund RA, Illes Z, Myhr KM, Torkildsen Ø. Recent progress in maintenance treatment of neuromyelitis optica spectrum disorder. J Neurol [Internet]. 2020;(0123456789) Available from. https://doi.org/10.1007/s00415-020-10235-5.

16. Axtell R, Raman $\mathrm{C}$, Lawrence $\mathrm{S}$. Interferon Beta exacerbates TH17 inflammatory diseases. Trends Immunol. 2011;32(6): 272-7.

17. Wynford-Thomas R, Jacob A, Tomassini V. Neurological update: MOG antibody disease. J Neurol [Internet]. 2019;266(5):1280-6. Available from:. https://doi.org/10.1007/s00415-018-9122-2.

18. Jarius S, Paul F, Aktas O, Asgari N, Dale RC, de Seze J, et al. MOG encephalomyelitis: international recommendations on diagnosis and antibody testing. Nervenarzt. 2018:1-10.

19. dos Passos GR, Oliveira LM, da Costa BK, Apostolos-Pereira SL, Callegaro D, Fujihara K, et al. MOG-IgG-associated optic neuritis, encephalitis, and myelitis: lessons learned from neuromyelitis optica spectrum disorder. Front Neurol. 2018;9(APR):1-10.

20. Reindl M, Waters P. Myelin oligodendrocyte glycoprotein antibodies in neurological disease. Nat Rev Neurol [Internet]. 2019;15(2): 89-102. Available from:. https://doi.org/10.1038/s41582-0180112-x.

21. Ramanathan S, Mohammad S, Tantsis E, Nguyen TK, Merheb V, Fung VSC, et al. Clinical course, therapeutic responses and outcomes in relapsing MOG antibody-associated demyelination. J Neurol Neurosurg Psychiatry. 2018;89(2):127-37.

22. Tenembaum SN. Acute disseminated encephalomyelitis. Handb Clin Neurol. 2013;112:1253-62.

23. Kunchok A, Zekeridou A, McKeon A. Autoimmune glial fibrillary acidic protein astrocytopathy. Curr Opin Neurol. 2019;32(3):452-8.

24. Hampton CW, Galperin D, Alvarez E, West MS. Erratum: Encephalomyeloneuropathy with CRMP-5 antibodies in a patient with a primary mediastinal serinoma (Neurology: Neuroimmunology and NeuroInflammation (2015) 2 (e82)). Neurol Neuroimmunol NeuroInflamm. 2015;2(4):1.

25. Kunchok A, Zekeridou A, Pittock S. CRMP5-IgG-associated paraneoplastic myelopathy with PD-L1 inhibitor therapy. JAMA Neurol. 2020;77(2):255-6.

26. Eurelings LEM, Miedema JR, Dalm VASH, Van Daele PLA, Van Hagen PM, Van Laar JAM, et al. Sensitivity and specificity of serum soluble interleukin-2 receptor for diagnosing sarcoidosis in a population of patients suspected of sarcoidosis. PLoS One [Internet]. 2019;14(10):1-15. Available from:. https://doi.org/10. 1371/journal.pone.0223897.
27. De Virgilio A, de Vincentiis M, Inghilleri M, Fabrini G, Conte M, Gallo A, et al. Idiopathic hypertrophic pachymeningitis: an autoimmune IgG4-related disease. Immunol Res. 2017;65(1): 386-94.

28. Detlefsen S, Klöppel G. IgG4-related disease: with emphasis on the biopsy diagnosis of autoimmune pancreatitis and sclerosing cholangitis. Virchows Arch. 2018;472(4):545-56.

29. Mehta SH. Clinical / Scientific Notes. 540-3.

30. Baptista B, Casian A, Mrcp MA, Rice CM, Uk M. Neurological manifestations of IgG4-related disease opinion statement pathophysiology. 2017;1-17.

31. Rumalla K, Smith KA, Arnold PM (2017). Immunoglobulin G4 related epidural inflammatory pseudotumor presenting with pulmonary complications and spinal cord compression: case report. 26(June) 688-693.

32. Al Sawaf A, Berger JR. Longitudinally extensive transverse myelitis suspected for isolated Neuro-Behçet: a diagnostic conundrum. Mult Scler Relat Disord. 2015;4(5):395-9.

33. Leccese P, Alpsoy E. Behçet's disease: an overview of etiopathogenesis. Front Immunol. 2019;10(MAY).

34. Yelnik CM, Kozora E, Appenzeller S. Non-stroke central neurologic manifestations in antiphospholipid syndrome. Curr Rheumatol Rep. 2016;18(2):1-9.

35. Gu S, Liu Y, Song Z, Zi X, Deng H. Acute myelitis in a patient with Vogt-Koyanagi-Harada disease: case report and review of the literature. J Clin Neurol. 2013;9(1):61-4.

36. Faissner S, Lukas C, Reinacher-Schick A, Tannapfel A, Gold R, Kleiter I. Amphiphysin-positive paraneoplastic myelitis and stiffperson syndrome. Neurol Neuroimmunol NeuroInflamm. 2016;3(6):1-3.

37. Llamas Y, Hazel K, Nicholson P, Costelloe L. Longitudinally extensive transverse myelitis after Campylobacter jejuni enteritis. Pract Neurol. 2018;18(2):143-5.

38. Neri VC, Xavier MF, Barros PO, Bento CM, Marignier R, Alvarenga RP. Case report: acute transverse myelitis after Zika virus infection. Am J Trop Med Hyg. 2018;99(6):141921.

39. Leone A, Marino M, Dell'Atti C, Zecchi V, Magarelli N, Colosimo C. Spinal fractures in patients with ankylosing spondylitis. Rheumatol Int. 2016;36(10):1335-46.

40. Isogai N, Asamoto S, Nakamura S, Sakurai K, Ishihara S, Ishikawa $\mathrm{M}$, et al. Spine and spinal cord injury associated with a fracture in elderly patients with ankylosing spondylitis. Neurol Med Chir (Tokyo). 2018;58(3):103-9.

41. Ulutatar F, Unal-Ulutatar C, Duruoz MT. Cervical proprioceptive impairment in patients with rheumatoid arthritis. Rheumatol Int [Internet]. 2019;39(12):2043-51. Available from. https://doi.org/ 10.1007/s00296-019-04419-0.

42. Chung J, Bak KH, Yi HJ, Chun HJ, Ryu J II, Han MH. Upper cervical subluxation and cervicomedullary junction compression in patients with rheumatoid arthritis. J Korean Neurosurg Soc. 2019;62(6):661-70.

43. Lee JJY, Schneider R. Systemic juvenile idiopathic arthritis. Pediatr Clin North Am [Internet]. 2018;65(4):691-709. Available from. https://doi.org/10.1016/j.pcl.2018.04.005.

44. Munz M, Wessendorf S, Koretsis G, Tewald F, Baegi R, Krämer S, et al. Acute transverse myelitis after COVID-19 pneumonia. J Neurol. 2020;26:1.

45. Durrani M, Kucharski K, Smith Z, Fien S. Acute transverse myelitis secondary to severe acute respiratory syndrome coronavirus 2 (SARS-CoV-2): a case report. Clin Pract Cases Emerg Med. 2020;4(3):344-8.

46. Chow CC, Magnussen J, Ip J, Su Y. Acute transverse myelitis in COVID-19 infection. BMJ Case Rep. 2020;13(8) e236720. 
47. Kaur H, Mason JA, Bajracharya M, McGee J, Gunderson MD, Hart BL, et al. Transverse myelitis in a child with Covid-19. Pediatr Neurol. 2020;1.

48. Valiuddin H, Skwirsk B, Paz-Arabo P. Acute transverse myelitis associated with SARS-CoV-2: a case-report. Brain Behav Immun Health. 2020.
49. Baghbanian SM, Namazi F. Post COVID-19 longitudinally extensive transverse myelitis (LETM)-a case report. Acta Neurol Belg. 2020;18:1-2.

Publisher's Note Springer Nature remains neutral with regard to jurisdictional claims in published maps and institutional affiliations. 